\title{
Information literacy: learning to use sources of information
}

TAMI TSOUK AND IRENE SEVER

Haifa University Library, Laboratory for Children's Librarianship

\begin{abstract}
Teaching primary school children skills of information retrieval is the beginning of a process of acquiring information literacy so necessary for an adequate mastery of present day information explosion. The program elaborated and applied in the Laboratory for Children's Librarianship of Haifa University Library aims at discovering what are the difficulties primary school pupils have in using bibliographic material and at creating means of coping with these difficulties. The subjects of the program are second to fourth graders from a variety of schools in Haifa area. The program is in its third year and has up to now encompassed over five hundred children.
\end{abstract}

\section{Research setting}

The Laboratory operates on the basis of five consecutive weekly visits of each class for an entire school day. The Laboratory represents a small library, containing some 12,000 books in Hebrew and 3,000 books in Arabic. The collection contains most of the encyclopedias and other reference materials available commercially. The laboratory has a one-way mirror and closed circuit television cameras for recording and uses eye-to-eye observations whenever suitable manpower is available. The staff of the lab consists of the teacher who carries out the different programs during the day, two researchers and the librarian in charge of the library. The time devoted to bibliographic instruction is divided into two sessions, each approximately 20 minutes long, the two sessions being separated by other activities such as free reading, snack break and story telling. The children work both in groups and individually, so that no one is left behind. All the children in the groups have a finished product of their work by the time of the final meeting, when it is presented to the class and whenever possible typed and bound together to be kept in the school's library.

\section{Research method}

The data collection is naturalistic and our goal is to identify the skills needed to be imparted for a successful completion of a research "project" by the children and to devise the means of facilitating the acquisition of those particular skills. The method of naturalistic observations of overt behavior enables us to take into consideration not only the difficulties of the entire class or of children of a particular age in general, but also the idiosyncratic problems of specific children. The data collected by observation and videotape serve to initiate alternative ways of imparting the information or skill that seemed to create

Education for All: Culture, Reading and Information, IASL, 1998 
difficulties. In this sense, the research project is an ongoing process of integrating findings into the program.

After an initial phase of observing children in action we found it useful to adapt some of the underlying principles of the Information Problem Solving: The Big Six Skills approach to library information skills instruction by Eisenberg and Berkowitz (1995) Obviously a great deal of adaptation must be made to this model before it can be used for our aims, our particular type of school system and its approach to information retrieval and subject matter.

Several research questions arise in this study:

1) What are the difficulties encountered by young children in retrieving and using information?

2) What is the process by which children acquire information from available sources?

3) What are the problems raised by the Big Six overall scheme that are specific to Israeli conditions? In what way does the systems of instruction used by the educational establishment affect the abilities of second to fourth graders in using information?

\section{The activity}

The program aims at balancing activities with the participation of the entire class, groups of four or five pupils and individual work.

Phase one deals with defining the general subject to be researched, subordinate subjects within a larger context and the specific question of each pupil within this framework. The formulation of the questions on the level of the individual, the group and the class is defined and decided upon.

Phase two aims at training the children to distinguish between questions that can be answered with the help of bibliographic materials at their disposal and those that cannot easily be answered.

Phases three and four are preparations for the final product: finding the appropriate materials, retrieving the essentials and discarding the unimportant or irrelevant.

Phase five is the preparation of the final product and the presentation of the individual contributions to the entire class.

The emphasis we wish to make in this paper is less to describe the process of teaching the skills required for this type of a task but rather to concentrate on what the observations revealed as difficulties encountered by the children in the course of their work. These difficulties we are attempting to counter by appropriate exercises so as to help the children overcome them and succeed in their appointed task.

Formulation of a question is understandably difficult. The problem lies in their naive assumption that the answer to ANY QUESTION can be found if looked for. The need to formulate the question in a manner that is answerable through the written materials available presupposes that the children have a knowledge of what encyclopedias and other reference sources can and cannot do. Therefore one of the first steps in finding answers to questions must be acquaintance with the tools and with the manner in which they are arranged and organise knowledge. Beyond this are answers that exists but are too 
difficult for the children to understand either because their presentation in the material is too difficult for a particular age group or because the particular scientific material is beyond the ability of the children to comprehend at their stage of development and knowledge. Sometimes the problem is that the material is not interesting and rewarding enough to justify the process of retrieval.

Sources of information and their categorization is another stepping stone on the way to knowledge. Adults tend to organize and categorize reference works according to more or less defined scientific classifications. They have no difficulty in differentiating between alphabetical and subject encyclopedias, and have no trouble in assigning data of information to the appropriate subject category or sub-category. Children on the other hand understandably lack this acquired framework and tend to categorize according to other criteria. At school they are mostly acquainted with alphabetical encyclopedias, to which they are referred by their teachers. The children little or no experience with subject encyclopedias which they often consider not encyclopedias at all, but rather as peculiarly organized books. They also tend to have difficulties in finding the relevant material within the the various volumes of the subject encyclopedia and their ability to locate a certain letter within an alphabetical encyclopedia is often shaky.

Techniques of searching for information must be developed. Children lack the ability to decide where the appropriate material may best be found, and are trained to deal with precise answers to precise questions. Devising their own way of looking up information requires special training as otherwise if the question cannot be answered by a direct quote from the text, the children were found to be often unable to present the information.

Evaluation of information presents a difficulty; the factual information to be found in encyclopedias, even those intended for young children is partly irrelevant to a specifically formulated question. Children tend to accept $A L L$ information unless the less relevant or even the irrelevant is pointed out. The accuracy of the information and its applicability to the question asked must be clarified, as without this any abstracting or extracting of information from a written text is impossible. Children unskilled in this tend to copy verbatim the first section of an encyclopedic entry even if it has only tenuous connection with the question asked.

The integration of the information into an understandable whole is another difficulty for children: the answer to a question must be created from information collected from several different sources or from different parts of the same source. Often the very act of finding the information causes the question to be forgotten and the data retrieved from the sources are no, ore connected to the original query.

Presentation of the material is not only dependent on the writing skill of each individual child but also on the ability to arrange the material in a logical format. Children seem to have difficulty in understanding that in a $Q$ and $A$ situation, the answer is often composed of a number of items of information. The hierarchy and internal composition of the various facets of the question and the answer to it must be explained before a lucid and relevant answer emerges. Therefore, the final product of any investigation made by the children must be a coherent and full description of the relevant bit of information retrieved. This has to be impressed on the students as as important as the search for the answer. 
Feedback from the search for an answer and use of the information acquired presents a difficulty. The children seem to find it hard to draw conclusions from their search for an answer even though they have fully understood the material and have seemingly absorbed the information. This last stage of assimilating the information gained may be a crucial stage in information literacy.

An analysis of our observations over a period of three years tends to show that the formulation of a question can be of different types: some questions can be best described as "emotional", for example: "why are dinosaurs frightening?" Obviously the answer to such a question can be found in encyclopedias, but only by inference: they are big, they look fierce, they are carnivores, etc. More often than not, the questions are too general to be answered properly by a single entry in an encyclopedia: for example: "I want to know about space and space travel." The opposite also occurs frequently, a question so specific that no bibliographic source can give a satisfactory answer: "who used this (prehistoric) utensil?" "who was the first person to do so and so?" A third type of unanswerable question is a qualitative, attitudinal question: "who is the best (painter, football or basketball player) in the world?" "Is a lion braver than an elephant?"

In order to counteract this tendency we have evolved a variety of means. One is asking the children, first by the entire class as an example and later in small groups to write down their associative thoughts with a given subject. Second-and third-graders are often interested in the animals or countries of the world. Writing down anything that comes into mind around a certain subject causes the children to think in increasingly larger contexts: they begin by listing all the animals or countries that they know, but if encouraged they begin to add hierarchically different aspects of the problem: instead of countries they begin to list such concepts as oceans, continents and rivers, for animals they begin to think in terms of habitats, and attributes. To encourage this trend we often introduce them to the subject around a story: an unknown animal has come to the zoo and nobody really knows how to take care of it, or we are planning a trip abroad to a certain country, what should we pack? This causes our little friends to think in terms of precise subdivisions of the subject and to reinforce this we hand out prepared pages that are subdivided into different aspects of the question: this has a twofold effect: one, the children stop looking for the answer to their question in the first paragraph of the entry in the reference book, and two, they learn that the answer must not necessarily be listed in the order it appears in the entry but rather that the different aspects of the answer can be found in different places and does not have to be entered into the answering sheet in any specific order.

Finding the correct information and extracting it from the correct volume, the correct entry or the correct part of the entry must be drilled : repeatedly we have observed children leafing through a volume of an alphabetical encyclopedia vainly hoping that the desired answer will leap to their eye from the pages of the book. The idea that a specific answer is best looked for in a specific source is not necessarily obvious to our young visitors. Many of them have alphabetic encyclopedias at home, and once they have solved the problem of what letter to search under, they revert to the familiar trend of copying whatever is written in the text, whether it is relevant or not. In order to counteract this tendency we write out on long strips of paper an entire entry, exhibit it on the blackboard, hand the children scissors and tell them to cut up the sentences into relevant and irrelevant material. What 
they feel is relevant goes into keeping in a safe, the other snips of sentences go into a wastepaper basket. Done by the entire class the first time, there is a lot of discussion as to the relative value of the snips. However, we seem to be able to get the idea across that all that is written in an encyclopedic entry is not necessarily indispensable to the question asked and that there are snips of information that may be important in another context but not in the present one.

This leads naturally into dealing with the question the children are dealing with: now it is more evident to them how the question: what should we feed that mysterious animal in the zoo or should we pack galoshes for a visit to Greenland, ought to be approached. The contents of the safe begins to make sense. Further we again put the whole text together and ask the class:

"What questions does this text answer. By having the children elicit the question from the text, we show them how sometimes new questions can arise from the text itself during the process of information retrieval. We try to point out that this process is never a linear, step-by-step process. This helps the children with another difficulty: changing the original (sometimes unanswerable) question with one that can be dealt with through the text.

Another drill is needed to explain the hierarchical nature of a question and the information about it. What an animal eats or where it lives cannot be deduced from its general appearance. It has to be clarified on the basis of some specific features: its teeth, its limbs, its coloring. In spite of the fact that most Israeli children are drilled in describing pictures in nursery school, we found that they have a difficulty in drawing conclusions from pictures. When we refer the children to the pictures in the encyclopedias, which nowadays are very explicit, beautiful and precise, what we get is plaintive cries: "it does not say here (in the text) "The fact that the animal is depicted as sitting in a tree munching leaves does not seem at first to elicit the information that a) the animal is arboreal and b) it is a vegetarian. But eventually the children seem to grasp that pictures are mines of information and once this bridge has been crossed they find pictures and illustrations very informative and as a rule after a drill or two they are able to extract a great deal of correct and relevant information from pictorial material.

Now comes the really hard part: put all the information together into an answer to the original questions. Just as adults, children are often sidetracked by all the material they have looked at and the original query has faded into insignificance compared to all the information gathered and discarded. By keeping the original question firmly in front of the children and with the help of the story that elicited it (the mysterious arrival in the zoo or the climate of Greenland) we expect the children to do two things: one, to check whether what they have written in the squares on the answering sheet is in fact the whole answer to the question they asked or perhaps the question that arose from the fact-finding process, and two, to present, verbally, aloud, to the entire class what information they have collected. The children presenting information about an animal or a country must describe the findings in their own words, not in the words of their encyclopedia, something many find difficult to do, but they must also decide who else in the class fits into the same category. For example the child describing a lion as living in the forest will find that a whale is not a suitable companion, he or she must look for a land animal living in the savanna. For example a giraffe lives in an habitat similar to that of the lion, but feeds on different materials and differs from the lion in other aspects. Each child is asked to describe in a few 
words his or her animal or country, draw its picture (or have it copied from a book and pasted) and then can wander around the lab looking for likely companions. The pieces of cardboard representing pizza wedges miraculously fit together and some discover that their particular pizza piece fits more than one finished pizza, while others may only create half a pizza and can be proud of the fact that their selection is special and exclusive.

The final product is of course the cumulative result of all that the children found in the course of their inquiry. In the case of second graders, they need parental or school help in typing their product. For third and fourth graders, part of the fun is preparing their final traveling guide or animal book to be proudly displayed in their class and later included in the school library.

The whole process, from start to finish takes no more than two sessions of about 20 minutes each in the course of 5 weekly visits in the lab. In terms of time, less than four hours. The fun and the pride in the end product are certainly justified.

\section{References}

Jansen, Barbare (1996). Reading for information: the trash-and-treasure method of teaching notetaking. School Library Activities Monthly, 12, 29-32.

Eisenberg, Michael and Robert Berkowitz (1995). Information problem solving. The big six skills approach to library and information skills instruction. Norwood, N.J.: Ablex.

Moore, Penelope and Alison St. George. (1991). Children as information seekers. School Library Media Quarterly, 19(3), 161-168.

Underwood, Jean and Geoffrey Underwood (1987). Data organisation and retrieval by children. British Journal of Educational Psychology, 57, 313-129. 